\title{
Consórcios Intermunicipais para o Desenvolvimento: Determinantes da Cooperação entre os Municípios Baianos
}

Ferreira Doin, Tatiana Aparecida; Morais Silva Lopes, Laerson; Souza Afonso, Adelzira; Scalfoni Rigo, Ariádne

Consórcios Intermunicipais para o Desenvolvimento: Determinantes da Cooperação entre os Municípios Baianos Administração Pública e Gestão Social, vol. 12, núm. 1, 2020

Universidade Federal de Viçosa, Brasil

Disponible en: http://www.redalyc.org/articulo.oa?id=351561601012

Esta obra está bajo una Licencia Creative Commons Atribución-NoComercial-SinDerivar 3.0 Internacional. 


\title{
Consórcios Intermunicipais para o Desenvolvimento: Determinantes da Cooperação entre os Municípios Baianos
}

\author{
Intermunicipal Consortia for Development: Determinants of Cooperation between the Municipalities of Bahia \\ Consorcios Intermunicipales para el Desarrollo: Determinantes de la Cooperación entre los Municipios Baianos \\ Tatiana Aparecida Ferreira Doin \\ Redalyc: http://www.redalyc.org/articulo.oa? \\ Universidade Federal da Bahia, Brasil \\ $\mathrm{id}=351561601012$
}

tatianadoin@hotmail.com

Laerson Morais Silva Lopes

Universidade Federal da Babia, Brasil

laerson.lopes@ufba.br

Adelzira Souza Afonso

Faculdade Estácio, Brasil

adelzira@yahoo.com.br

Ariádne Scalfoni Rigo

Universidade Federal da Bahia, Brasil

ariadne.rigo@ufba.br

Recepción: 20 Diciembre 2017

Aprobación: 19 Agosto 2018

Publicación: 01 Enero 2020

\section{Resumo:}

O presente artigo se propõe a identificar quais características econômicas, fiscais, demográficas e geográficas dos municípios baianos influenciam na formação de consórcios intermunicipais de desenvolvimento. A fundamentação teórica do estudo baseou-se na Teoria da Ação Coletiva Institucional, importante lente adotada na literatura para a análise dos fatores que condicionam a propensão à cooperação intergovernamental. Quanto ao método, o estudo é descritivo e exploratório, com abordagem quantitativa, que utilizou bases de dados secundárias para analisar, pela estatística inferencial e o modelo de regressão logística, os dados coletados dos 417 municípios baianos. Os resultados evidenciaram três características correlacionadas, ou seja, que influenciam a formação de consórcios intermunicipais de desenvolvimento: Maior receita tributária, maior porte (população) e municípios inseridos em aglomeração urbana. Sugere-se que estudos futuros analisem a economia de escala decorrente destes acordos e avaliem a influência da orientação política na cooperação intermunicipal.

PALAVRAS-CHAVE: ação coletiva institucional, cooperação intergovernamental, gestão municipal.

\section{Abstract:}

This article proposes to identify the economic, fiscal, demographic and geographical characteristics of the municipalities of the state of Bahia, in Brazil, which influence the formation of intermunicipal development consortium. The theoretical foundation of the study was based on the Theory of Institutional Collective Action, important lens adopted in the literature for analysis of the factors that condition the intergovernmental cooperation. As for the method, the study is descriptive and exploratory, with a quantitative approach, which uses secondary databases to analyze, through inferential statistics and logistic regression model, the collected data of the 417 municipalities of Bahia. The results showed three characteristics that influence the formation of intermunicipal consortia for development: Bigger tax revenue, bigger population and municipalities inserted in urban agglomeration. In new research, it is suggested an analysis of the economy of scale and an evaluation of the political orientation in the influence in the intermunicipal cooperation.

KEYWORDS: Institutional Collective Action, intergovernamental cooperation, municipal management.

\section{Resumen:}


El presente artículo se propone identificar las características económicas, fiscales, demográficas y geográficas de los municipios baianos que influencian la formación de consorcios intermunicipales de desarrollo. La fundamentación teórica del estudio se basa en la Teoría de la Acción Colectiva Institucional, lente importante adoptada en la literatura para el análisis de los factores que condicionan la propensión hacia la cooperación intergubernamental. En relación al método, el estudio es de carácter descriptivo y exploratorio, de enfoque cuantitativo, que utilizó bases de datos secundarias para analizar por estatística inferencial y el modelo de regresión logística, los datos colectados de los 417 municipios baianos. Los resultados evidenciaron tres características corelacionadas, es decir, que influencian la formación de consorcios intermunicipales de desarrollo: Mayor recaudo tributario, mayor porte (población) y municipios inseridos en aglomeración urbana. Se sugiere que estudios futuros analicen la economía de escala resultante de esos acuerdos y evaluen la influencia de la orientación política en la cooperación intermunicipal.

Palabras Clave: acción colectiva institucional, cooperación intergubernamental, gestión municipal.

\section{INTRODUÇÃO}

No Brasil, os governos, em particular os dos municípios, enfrentam dificuldades diante da série de obrigações que precisam cumprir e das limitações de recursos para tal. Estes desafios, inerentes ao desequilíbrio do modelo de federalismo instituído, podem ser equacionados, segundo Randolph e Magalhães (2017), pela criação de instrumentos de coordenação e cooperação. A cooperação federativa é um desses instrumentos, tanto que mais de 3.100 municípios do país já recorreram a ela como uma das alternativas de gestão (Linhares, Messenberg \& Ferreira, 2017). Esta é uma estratégia de mobilização de entes de um determinado território que envolve o diagnóstico de um problema comum e o levantamento de custos e insumos necessários para solucioná-lo, considerando quanto cada ente pode compartilhar e oferecer para solucionar o problema dos recursos (Cruz, Araújo \& Batista, 2011).

Quanto à forma, a cooperação pode ser do tipo técnica e financeira e de estrutura vertical ou horizontal. Enquanto a vertical compreende o compartilhamento de ações e soma de esforços de uma política pública (cooperação intergovernamental) ou de políticas públicas (intragovernamental) entre as diversas esferas de governo, a cooperação horizontal compreende ações e soma de esforços de diferentes setores e agências em uma mesma esfera de governo (intragovernamental) ou de entes de uma mesma esfera de governo (intragovernamental) (Farah \& Jacobi, 2000). Dentre estas formas, o consórcio público é considerado uma inovação do federalismo brasileiro (Grin, Segatto \& Abrucio, 2016).

No âmbito da cooperação horizontal, os consórcios públicos podem ser internacionais, interestaduais ou intermunicipais. $\mathrm{O}$ objeto de estudo deste artigo são os consórcios públicos intermunicipais. O consórcio público intermunicipal, exemplo de prática inovadora, também busca reunir as potencialidades do local, para dar conta dos problemas enfrentados, que não são resolvidos via instâncias mais amplas. Neste aspecto, o local é um espaço importante e valorizado para inovações de gestão pública (Teixeira, 1999).

Observa-se, em relação ao consórcio público intermunicipal, que, embora este seja capaz de distribuir benefícios entre os municípios consorciados, existem custos associados que são compartilhados e nem sempre há uma percepção equânime em relação aos ganhos obtidos (Machado \& Andrade, 2014). Por exemplo, temse a redução dos problemas relacionados aos efeitos de transbordamento entre municípios de maior porte e municípios de menor porte com comportamento free rider (carona). $\mathrm{O}$ efeito transbordamento geralmente ocorre entre municípios vizinhos quando um município de menor porte e com insuficiência de recursos se beneficia de produtos e serviços oferecidos por um município maior sem absorver os custos, "um clássico dilema da ação coletiva" (Machado \& Andrade, 2014, p. 700).

Visando compreender estas questões de ação coletiva entre governos, Feiock (2007) desenvolveu uma abordagem teórica denominada Ação Coletiva Institucional, na qual identificou características do governo local que influenciam a cooperação intermunicipal, classificando-as como exógenas e endógenas. Seguindo essa linha, Mazzali e Niero (2015) analisaram algumas dessas características relacionadas aos fatores endógenos que favorecem a constituição de arranjos cooperativos em governos locais no estado de São Paulo. Dentre elas, os autores identificaram que a dependência de recursos fiscais de esferas superiores e a inserção 
do município em aglomeração urbana motivam a cooperação, ao passo que os custos de transação configuram uma barreira. Porém, os autores não confirmaram a relação entre a realização de arranjos colaborativos e características como a receita tributária, a dívida fundada, a condição econômica e o porte do município.

Ainda que a pesquisa de Mazzali e Niero (2015) tenha sido seminal no Brasil, estabelecendo relação entre os fatores endógenos e os consórcios públicos utilizando a abordagem da Ação Coletiva Institucional, a heterogeneidade das características do território brasileiro requer uma análise sistemática desta lacuna empírica nos demais estados e municípios. Ademais, alguns achados destes autores vão de encontro ao que prevê a literatura internacional. Nesse sentido, o objetivo deste artigo é identificar as características econômicas, fiscais, demográficas e geográficas dos municípios baianos que influenciam a formação de consórcios intermunicipais de desenvolvimento.

Os estudos brasileiros sobre consórcios públicos geralmente são qualitativos, majoritariamente de estudos de caso (Abrucio, Filippim \& Dieguez, 2014; Afonso, Vieira, Santos Junior \& Doin, 2016; Galindo, Cordeiro, Villani, Barbosa \& Rodrigues, 2014; Machado \& Palotti, 2015; Nascimento \& Fernandes, 2015). Embora esse tipo de estudo tenha suas contribuições, a metodologia empregada dificulta a generalização dos resultados e respectiva teorização da temática (Mazzali \& Niero, 2 015). Poucos são os estudos que se assentam na análise quantitativa da cooperação intermunicipal no Brasil. Numa consulta realizada em março de 2017 no Google Scholar foram identificados somente três artigos publicados em periódicos nacionais que privilegiaram os métodos quantitativos (Mazzali \& Niero, 2015; Pereira \& Moreira, 2016a, 20 16b).

Por outro lado, a prática demonstra o crescimento no número de consórcios públicos no país. De acordo com os dados da Pesquisa de Informações Básicas Municipais (MUNIC) sobre o perfil dos municípios brasileiros, desenvolvida pelo Instituto Brasileiro de Geografia e Estatística (IBGE) em 2015, 66,3\% dos municípios brasileiros possuem algum tipo de consórcio. Quanto à cooperação interlocal, 96,7\% dos municípios constituíram consórcios intermunicipais, sendo observado um aumento considerável na cooperação horizontal em detrimento à cooperação vertical num comparativo entre os anos de 2011 e 2015 (IBGE, 2015).

Portanto, dado o crescimento deste tipo de arranjo colaborativo como uma estratégia ao enfrentamento dos desafios da esfera municipal e a importância dos estudos sobre cooperação interlocal na contemporaneidade, esta pesquisa mostra-se relevante por oferecer um panorama sobre as características que influenciam a cooperação via consórcios públicos de desenvolvimento da Bahia. A escolha por consórcios de desenvolvimento deve-se à quantidade desses arranjos instituídos no estado, em função de uma política do governo estadual para promover o desenvolvimento dos territórios de identidade, que, a partir de 2005, aumenta consideravelmente o número desses (Carvalho, Xavier \& Pinto, 2016).

Ressalta-se também a contribuição para a adoção da análise quantitativa enquanto método de pesquisa no Brasil para a observação das características locais, seguindo a tendência da literatura internacional (Bel \& Warner, 2016). Desse modo, o artigo é capaz de ampliar o horizonte de análise para o nível macro da constituição de consórcios públicos intermunicipais de desenvolvimento.

\section{REFERENCIAL TEÓRICO}

\subsection{O federalismo brasileiro e a cooperação intergovernamental}

As peculiaridades em termos de heterogeneidade demarcam o modelo federativo brasileiro (Grin et al., 2016). O federalismo é uma forma de organização de Estado na qual os entes federados são dotados de autonomia administrativa, política, tributária e financeira, necessárias para manter o equilíbrio que se estabelece entre eles para a constituição do Estado Federal (Oliveira, 1995). Portanto, ele parte do pressuposto de que deve 
haver repartição de responsabilidades governamentais de modo a assegurar a integridade do Estado nacional frente às inúmeras disputas e desigualdades regionais.

O arcabouço institucional do federalismo brasileiro foi delineado inicialmente pela primeira Constituição no ano de 1824, ainda no Brasil Império, culminando na sétima Carta Constitucional de 1988, nas quais podem ser observados avanços e retrocessos em relação à descentralização e autonomia dos entes federados (Abrucio, 2005; Abrucio \& Sano, 2012). Neste interstício, Prates (2012) observou que desde a Constituição Federal de 1891 já havia a previsão legal de arranjos de cooperação entre entes federativos, constituindo consórcios sob a forma de contratos. Do mesmo modo, traçando uma linha temporal, Machado e Andrade (2014) demonstram como ocorreu a evolução deste processo normativo, constatando como é longínqua a preocupação do Estado brasileiro com as possibilidades de cooperação intermunicipal, vindo desde a Segunda República até o Período Democrático recente, com a promulgação do Decreto n ${ }^{\circ} 6.017$, de 17 de janeiro de 2007.

As variadas formas de cooperação podem contribuir para reduzir as assimetrias, conflitos e tensões provocados pelas especificidades do federalismo versus as disparidades do território brasileiro. Desse modo, o consórcio público, enquanto uma forma de cooperação, é conceituado como

[...] pessoa jurídica formada exclusivamente por entes da Federação, na forma da Lei no 11.107, de 2005, para estabelecer relações de cooperação federativa, inclusive a realização de objetivos de interesse comum, constituída como associação pública, com personalidade jurídica de direito público e natureza autárquica, ou como pessoa jurídica de direito privado sem fins econômicos (art. 2. ${ }^{\circ}$, inc. I, Decreto n. ${ }^{\circ}$ 6.017/2007)

Os consórcios públicos podem ser uma forma de superar a inexistência ou precariedade dos mecanismos de coordenação de políticas públicas de responsabilidade partilhada entre o Governo Federal, os estados e os municípios. Não obstante, Cunha (2004, p. 40) faz uma importante ponderação sobre essa forma de cooperação: Embora os consórcios públicos possam "apoiar a execução cooperada de políticas com conteúdos de transversalidade e territorialidade", ainda não são capazes, por si só, "de resolver todos os conflitos e pendências do desenho ainda inconcluso da federação brasileira".

Segundo Andrew (2009, p. 140), "o objetivo mais amplo de acordos de cooperação para governos locais pode ser aumentar a prestação de serviços urbanos por meio da melhoria da prestação de serviços, mantendo ou reduzindo custos". Ou seja, essa cooperação, além de buscar a melhoria da prestação de serviços públicos, visa obter economias de escala e reduzir os impactos das restrições orçamentárias (Bel \& Warner, 2016).

No entanto, para que a cooperação intermunicipal ocorra, é necessário que haja preferencialmente um consenso em relação aos interesses comuns, aos riscos associados às pressões político-eleitorais, às regras, ao rateio dos custos de produção e de prestação de serviços, assim como os demais custos de transação e fatores contextuais associados aos problemas da ação coletiva (Feiock, 2007; Machado \& Andrade, 2014). Com vistas a elucidar os possíveis fatores que levam os agentes públicos a decidirem pela cooperação, na próxima seção serão detalhados os aspectos centrais da Teoria da Ação Coletiva Institucional.

\subsection{A perspectiva da Ação Coletiva Institucional}

A Teoria da Ação Coletiva Institucional é uma importante lente para a análise dos fatores que condicionam a propensão à cooperação intergovernamental (Feiock, 2007, 2013; Feiock \& Carr, 2001). Nesta perspectiva, Feiock (2007, p. 48) recupera a abordagem da segunda geração da teoria da escolha racional de Ostrom (2005) - a ação coletiva “[...] para a governança regional voluntária” -, sugerindo um conjunto de proposições com o intuito de explicar como surgem e evoluem arranjos descentralizados de governança voluntária. Com foco nas motivações e barreiras em relação aos custos de transação para esse tipo cooperação interlocal, a teoria tornouse um framework de análise em estudos empíricos, como o da cooperação no âmbito do desenvolvimento econômico local (Tavares \& Feiock, 2014), dentre outros. 
A Ação Coletiva Institucional é definida por Feiock (2007) como um conjunto de mecanismos pelo qual dois ou mais governos agem coletivamente para obter os ganhos decorrentes da prestação ou produção de serviços num perímetro maior. Para o autor, o interesse na participação em acordos de cooperação reside na combinação de fatores endógenos e exógenos. Do mesmo modo, Andrew (2009) indica que dentre as três abordagens de pesquisa predominantes que tratam da adoção de acordos de cooperação, a da ação coletiva institucional analisa os arranjos cooperativos para capturar as dimensões sociais (fatores endógenos) e dimensões estruturais (fatores exógenos), buscando explicar a adoção de acordos de cooperação interlocais e identificar a influência das diferentes estruturas nas motivações e barreiras para a constituição desse tipo de arranjo.

Os fatores exógenos referem-se às características econômicas, sociais, demográficas, geográficas e instituições políticas da comunidade (Feiock, 2007; Feiock \& Carr, 2001). Segundo Feiock (2007, p. 54), essas características "moldam as preferências por bens públicos e ajudam a determinar o potencial de ganhos e os custos de transação da cooperação". Dito de outro modo, os agentes públicos avaliam os custos e benefícios em relação à decisão de cooperar, como também as características da localidade, as características de um bem ou serviço e as configurações das instituições políticas vigentes.

Por outro lado, os fatores endógenos estão relacionados às redes de relações formais e informais formadas pelos agentes públicos de diferentes comunidades, sob as quais compartilham valores e normas de confiança e reciprocidade entre as partes (Feiock, 2007; Feiock \& Carr, 2001). Desse modo, quando a cooperação continua a fornecer benefícios aos participantes por intermédio destas redes, "as partes constituem reputações para que essas trocas sejam confiáveis, oferecendo no processo um mecanismo de feedback que aumenta a futura cooperação e ação coletiva" (Feiock, 2007, p. 60).

Assim, a análise da Ação Coletiva Institucional recai tanto sobre as motivações que orientam os agentes públicos em direção à cooperação intergovernamental quanto sobre as barreiras oriundas dos custos de transação de várias fontes como os custos de coordenação e informações para a identificação de potenciais ganhos na ação comum, os custos de negociação na divisão de ganhos mútuos, o monitoramento dos custos e divisão de atividades e os custos relacionados aos problemas de agência (Feiock, 2007).

Neste artigo, tal como Mazzali e Niero (2015), optou-se por analisar empiricamente os fatores exógenos e contextuais. Dentre esses fatores que influenciam a cooperação entre os governos locais estão as condições fiscais e econômicas dos municípios, como também as características demográficas e geográficas. Essas características são comumente identificadas na literatura sobre Ação Coletiva Institucional.

As condições fiscais estão relacionadas a outros três fatores que limitam a ação dos gestores em seus municípios que, segundo Krueger e Mcguire (2005), podem vir a orientá-los em direção à cooperação interlocal na tentativa de obter algum ganho com essa interação: A insuficiência de recursos, o estresse fiscal e a dependência de recursos de esferas superiores. Corroborando com esse estudo, Bel e Warner (2016) consideram que as restrições fiscais, inicialmente, induziram governos locais à privatização e, posteriormente, à cooperação intergovernamental para reduzir custos. De acordo com os autores, as restrições fiscais têm sido operacionalizadas por diferentes variáveis na literatura internacional, dentre elas, a receita tributária per capita, o peso da dívida pública per capita (relacionado ao estresse fiscal), etc.

A insuficiência de recursos é oriunda de uma base tributária deficitária (ou seja, quando a receita tributária é insuficiente para cobrir as despesas orçamentárias) e pode levar os municípios a buscarem acordos de cooperação com municípios vizinhos (Leroux \& Carr, 2007; Krueger \& Mcguire, 2005). Desse modo, Krueger e Mcguire (2005) sugerem que municípios com uma carga tributária menor são mais propensos a optar pela colaboração. No caso brasileiro, geralmente os municípios que possuem menor receita tributária tendem a se consorciar (Pereira \& Moreira, 2016a), embora no estado de São Paulo isso não tenha se evidenciado (Mazzali \& Niero, 2015).

O estresse fiscal está relacionado a um fator que influencia a cooperação intermunicipal quando há uma alta carga tributária combinada à implementação de um grande volume de políticas públicas (Hawkins, 
2007). Somando-se a isso, Krueger e Mcguire (2005) identificam a cooperação intermunicipal como uma possibilidade de redução de custos e respectivos impostos, dada a elevada carga tributária. Benton (2013) sinaliza que o estresse fiscal e a tensão causada pela recessão parecem ter sido parcialmente responsáveis por uma retomada do interesse em cooperar por parte dos governos municipais. Cabe ressaltar que, no Brasil, a métrica considerada para o estresse fiscal é a dívida fundada a longo prazo. Todavia, em São Paulo não foi constatado que os municípios cooperam em função do estresse fiscal, contrariando os achados apontados pela literatura internacional (Mazzali \& Niero, 2015).

O grau de dependência de recursos em relação aos recursos fiscais de esferas superiores é derivado de quão dependente o município se torna em relação aos recursos de transferências intergovenamentais (Krueger \& Mcguire, 2005). No Brasil, geralmente, "os municípios de menor população possuem fraca receita tributária e dependem fortemente de recursos de transferências intergovernamentais, notadamente as federais, sejam as constitucionalmente estabelecidas ou as voluntárias" (Fernandes \& Araújo, 2015, p. 298). Portanto, a transferência de recursos de esferas superiores se apresenta como uma variável com significativo poder de explicação do aumento da cooperação municipal (Kruege \& Mcguire, 2005). Os achados de Mazzali e Niero (2015) confirmam esta tendência da associação entre o interesse em cooperar e o grau de dependência de esferas superiores, visto que em São Paulo a cooperação horizontal induzida (incentivada por outras esferas governamentais) foi um dos fatores que justificou as maiores transferências intergovernamentais nesse estado.

Conforme Mazzali e Niero (2015), além de a geração de recursos (condições fiscais) influenciar a motivação na cooperação intermunicipal, outros fatores podem ser determinantes, como as condições econômica e social das cidades. No tocante à condição econômica, Krueger e Mcguire (2005) observam que altas taxas de desemprego, pobreza ou uma demografia majoritariamente jovem impactam as condições econômicas das cidades. Logo, para os autores, os municípios com menores condiçóes econômicas tendem a buscar a cooperação como uma alternativa para obter recursos, no sentido de promover programas sociais, visando mitigar os problemas socioeconômicos. Para Feiock (2007), os municípios que enfrentam dificuldades econômicas são mais propensos a colaborar com municípios vizinhos em projetos comuns. No entanto, os estudos de Mazzali e Niero (2015) constataram que a condição econômica atrelada ao PIB per capita não motiva a cooperação intermunicipal de acordos de desenvolvimento. Em oposição ao proposto pela literatura internacional, no estado de São Paulo "quanto maior o PIB per capita do município, maior o interesse na participação em acordos de desenvolvimento" (Mazzali \& Niero, 2015, p. 81).

As características demográficas dos municípios também exercem influência na cooperação interlocal. Quanto ao porte dos municípios, o indicador é o número de habitantes. De acordo com Bel e Warner (2016), a população é a variável mais comum em estudos que buscam identificar a influência das economias de escala na cooperação intermunicipal. Hawkins (2007) afirma que municípios de grande porte possuem maior base tributária e acesso ao mercado de capitais, isso propicia a captação de recursos para a produção e oferta de serviços com menores custos operacionais, o que não ocorre com municípios de menor porte, tendo em vista o menor número de habitantes, a base tributária reduzida e a dificuldade de acesso ao mercado de capitais. Segundo Post (2004), uma forte motivação para os municípios de menor porte adotarem acordos de cooperação é a oportunidade de obter os benefícios das economias de escala. A autora ressalta, assim como Hawkins (2007), que os municípios de maior porte possuem maiores chances de obter economias de escala em relação aos de menor porte. Os resultados dos estudos brasileiros são discrepantes, ao passo que o estudo do Mazzali e Niero (2015) sobre o estado de São Paulo constatou que quanto maior o porte, maior o interesse na participação em acordos de desenvolvimento, enquanto no nível nacional, quanto menor o porte do município, maior a propensão à adoção de consórcios públicos (Pereira \& Moreira, 2016a).

No âmbito das características geográficas, a localização é um dos mais importantes fatores, pois as fronteiras geográficas podem influenciar na cooperação intermunicipal quando os custos de transação são elevados, para alcançar uma redução por meio das interdependências e percepção de problemas comuns (Feiock, 2007). No Brasil, essa percepção pode ser acentuada quando os municípios pertencem a aglomerações urbanas. 
No caso da aglomeração urbana, os municípios que pertencem a ela possuem maior probabilidade de formarem acordos de cooperação (Morgan \& Hirlinger, 1991). A alta densidade demográfica no entorno possibilita a identificação dos problemas comuns, o que pode gerar a necessidade de compartilhamento de recursos e uma propensão entre os municípios circunscritos a cooperarem (Morgan \& Hirlinger, 1991; Krueger \& Mcguire, 2005; Post, 2004). Além disso, os interesses econômicos e a necessidade de resolver problemas de externalidade fornecem às cidades em áreas metropolitanas fortes incentivos para agir coletivamente para atingir metas comuns ou regionais (Feiok, 2007). Bel e Garner (2016) observam ainda que acordos de cooperação entre municípios vizinhos, mesmo em subúrbios, para serviços multifinalitários (produção de múltiplos serviços) são especialmente importantes em regióes metropolitanas.

Por fim, o presente artigo se ampara na pesquisa de Mazzali e Niero (2015, p. 33), que a partir dos fatores exógenos da perspectiva da Ação Coletiva Institucional, elencou sete hipóteses para identificar "a influência das características econômicas, fiscais, demográficas, sociais e geográficas das comunidades locais na propensão a cooperar por parte dos seus governos, com o propósito de estimular/sustentar o desenvolvimento econômico" nos acordos de cooperação intermunicipal do estado de São Paulo. Ou seja, como o conjunto dessas características configura um contexto favorável à cooperação intermunicipal (Feiock, 2007). Desse modo, este artigo propõe seis hipóteses, baseadas nos resultados de Mazzali e Niero (2015), para analisar as características dos municípios que celebraram consórcios intermunicipais de desenvolvimento no contexto do estado da Bahia:

Hipótese 1: Quanto maior a base tributária, maior a motivação e interesse da comunidade para a formação de acordos interlocais;

Hipótese 2: Quanto menor o nível de estresse fiscal, maior a motivação e interesse da comunidade para a formação de acordos interlocais;

Hipótese 3: Quanto maior a dependência de recursos fiscais de esferas superiores, maior a motivação e interesse da comunidade para a formação de acordos interlocais;

Hipótese 4: Quanto mais alta a condição econômica, maior a motivação e interesse da comunidade para a formação de acordos interlocais;

Hipótese 5: Quanto maior o porte do município, maior a motivação e interesse da comunidade para a formação de acordos interlocais;

Hipótese 6: O pertencimento do município a uma aglomeração urbana eleva a percepção dos problemas comuns, por parte das comunidades, aumentando a motivação e interesse na formação de acordos interlocais.

\section{METODOLOGIA}

Este artigo resulta de uma pesquisa do tipo levantamento via bases de dados secundárias e se propõe a identificar quais as características econômicas, fiscais, demográficas e geográficas de municípios baianos que influenciam a formação de consórcios intermunicipais de desenvolvimento entre estes municípios.

Para a estatística inferencial, a variável dependente - no caso, motivação e interesse em cooperar - busca captar a participação de municípios baianos em consórcios intermunicipais de desenvolvimento. Para a identificação dos que participam desses consórcios, recorreu-se inicialmente à lista elaborada pela Secretaria de Planejamento do Estado da Bahia (SEPLAN), com os consórcios realizados até dezembro de 2013. A lista da SEPLAN (2013) com 28 consórcios apresenta, entre outros dados, o nome dos mesmos, o território de identidade e a relação dos municípios cooperantes (www.consorciospublicos.ba.gov.br/home.html).

Tais dados foram confrontados com os disponibilizados pela instituição Observatório dos Consórcios Públicos e do Federalismo (http://www.ocpf.org.br/category/consorcios-publicos/), vinculado à Frente Nacional de Prefeitos, para confirmar quais consórcios atuavam na área de desenvolvimento. Quando não foi possível confirmar por esse meio, recorreu-se também à consulta dos dados de atuação do consórcio pela 
ferramenta do Google . Com isso, foi identificado que dois dos consórcios atuavam especificamente na área de saúde, sendo estes excluídos da relação considerada neste estudo.

Esta relação também foi confrontada com os dados do relatório MUNIC 2015, do IBGE (2015), analisando se os municípios listados na primeira sinalizaram a esse instituto se haviam celebrado "Consórcio de desenvolvimento Intermunicipal”. Verificou-se que alguns dos municípios constantes na relação da SEPLAN não sinalizaram a participação em consórcios de desenvolvimento no relatório do IBGE (2015). Devido à identificação de algumas inconsistências na base do IBGE, no que diz respeito a alguns consórcios de municípios que não estavam na base, todavia, já haviam sido aprovados em 2013 por lei e estavam inseridos na lista da SEPLAN (2013). Desse modo, optou-se somente pela lista da SEPLAN (2013) para estabelecer a relação entre consórcios e municípios participantes (dado indispensável para o cálculo de uma das variáveis do estudo).

Portanto, a variável dependente considerou a relação de municípios participantes de consórcios conforme dados da SEPLAN (2013), sendo identificados pelo número "1" os municípios que participam de consórcios intermunicipais. Isso feito, foram incluídos na base todos os outros municípios baianos, o que permite afirmar que esta é uma pesquisa populacional. Assim sendo, a variável explicativa é não métrica, dicotômica, podendo assumir os valores de 0 (não coopera) e 1 (coopera). Considerando isso, a base de dados foi formada por 458 observações (a Bahia possui 417 municípios, mas alguns participam de mais de um consórcio, constando, assim, mais de uma vez na base de dados).

As variáveis independentes, conforme demonstrado na Figura 2, são: Base tributária, estresse fiscal, dependência de recursos fiscais de esferas superiores, condição econômica, porte do município e a última, não métrica, é a inserção em aglomeração urbana. A figura 1 explicita as dimensões teóricas, o referencial teórico de base das variáveis, o nome da variável e o respectivo indicador. 


\begin{tabular}{|c|c|c|c|}
\hline $\begin{array}{l}\text { Dimens ã } \\
\text { o teórica }\end{array}$ & $\begin{array}{c}\text { Referencial teórico da } \\
\text { variável }\end{array}$ & $\begin{array}{l}\text { Nome da } \\
\text { Variável }\end{array}$ & Indic ador \\
\hline \multirow{3}{*}{ Fiscais } & $\begin{array}{l}\text { (BEL; WARNER, 2016; } \\
\text { KRUEGER; MCGUIRE, } \\
\text { 2005; LEROUX; CARR, } \\
\text { 2007; PEREIRA; } \\
\text { MOREIRA, 2016b) }\end{array}$ & $\begin{array}{l}\text { Base } \\
\text { tributária }\end{array}$ & $\begin{array}{c}\text { Receita } \\
\text { tributária } \\
\text { municipal per } \\
\text { capita }\end{array}$ \\
\hline & $\begin{array}{l}\text { (BEL; WARNER, 2016; } \\
\text { BENTON, 2013; } \\
\text { HA WKINS, 2005; } \\
\text { KRUEGER; MCGUIRE; } \\
\text { 2005) }\end{array}$ & $\begin{array}{c}\text { Estresse } \\
\text { fiscal }\end{array}$ & $\begin{array}{c}\text { Divida } \\
\text { fundada a } \\
\text { longo prazo } \\
\text { per capita }\end{array}$ \\
\hline & $\begin{array}{l}\text { (FERNANDES; } \\
\text { ARAÚJO, 2015; } \\
\text { KRUEGER; MCGUIRE, } \\
\text { 2005; MAZZALI; } \\
\text { NIERO, 2015) }\end{array}$ & $\begin{array}{l}\text { Dependênci } \\
\text { a de } \\
\text { recursos }\end{array}$ & $\begin{array}{c}\text { Receita de } \\
\text { trans ferências } \\
\text { interg overnam } \\
\text { entais per } \\
\text { capita }\end{array}$ \\
\hline $\begin{array}{c}\text { Econômic } \\
\text { as }\end{array}$ & $\begin{array}{c}\text { (FEIOCK, 2007; } \\
\text { KRUEGER; MCGUIRE, } \\
\text { 2005) }\end{array}$ & $\begin{array}{l}\text { Condição } \\
\text { econômica }\end{array}$ & $\begin{array}{c}\text { Produto } \\
\text { intemo bruto } \\
\text { per capita }\end{array}$ \\
\hline $\begin{array}{l}\text { Demongr } \\
\text { áficas }\end{array}$ & $\begin{array}{c}\text { (BEL; WARNER, 2016; } \\
\text { MA ZZALI; NIERO; } \\
\text { 2015; PEREIRA; } \\
\text { MOREIRA, 2016a) }\end{array}$ & $\begin{array}{l}\text { Porte do } \\
\text { municipio }\end{array}$ & \begin{tabular}{|c|} 
Número de \\
habitantes do \\
Município em \\
2010 \\
\end{tabular} \\
\hline $\begin{array}{c}\text { Geográfi } \\
\text { cas }\end{array}$ & $\begin{array}{l}\text { (BEL; WARNER, 2016; } \\
\text { KRUEGER; MCGUIRE, } \\
\text { 2005; LEROUX; CARR, } \\
\text { 2007; PEREIRA; } \\
\text { MOREIRA, 2016b) }\end{array}$ & $\begin{array}{l}\text { Inserção em } \\
\text { aglomeração } \\
\text { urbana }\end{array}$ & $\begin{array}{c}1 \text { se o } \\
\text { município } \\
\text { integra } \\
\text { aglomeração } \\
\text { urbana, ou } 0 \\
\text { se não. }\end{array}$ \\
\hline
\end{tabular}

Figura 1 - Síntese das variáveis independentes Fonte: Elaborado pelos autores (2017).

Em relação às variáveis do tipo razão, os dados dos indicadores foram obtidos de forma direta das bases de dados secundárias como: Secretaria do Tesouro Nacional (STN-Siconfi) (2013) para as variáveis base tributária, estresse fiscal e dependência de recursos; Superintendência de Estudos Econômicos e Sociais da Bahia (SEI) (2014) para a variável condições econômicas; e IBGE (2010) para o porte do município. Contudo, no caso específico da variável inserção em aglomeração urbana, considerou-se a delimitação feita no relatório "Arranjos Populacionais e Concentrações Urbanas do Brasil” (IBGE, 2016; IPEA, 2013). Os municípios que, segundo o estudo do Instituto de Pesquisa Econômica Aplicada (IPEA), fazem parte de aglomerações urbanas e regióes metropolitanas, foram considerados como inseridos em aglomeração urbana. Dessa forma, a variável inserção em aglomeração urbana recebeu valor "1" para os municípios baianos que fazem parte de aglomerações urbanas e "0" para os que não pertencem a aglomerações.

Considerando que a variável dependente deste estudo possui escala nominal, binária, e que as variáveis independentes são métricas e apenas uma delas é nominal, o modelo estatístico adotado foi o da regressão logística, baseado em Fávero et al. (2009) e Field (2009). A equação para a análise é a que segue:

$$
P\left(\text { Coop }_{\text {Inter }}\right)=\frac{1}{1+e^{-\left(a+B A S E_{T R I B}+S T R E S S+D E P E N D_{F I S C}+C O N D_{E C O}+P O R T E+O A G L O M\right)}}
$$


Onde:

Coop_Inter : Variável dependente, dicotômica, que indica se o município coopera (1) ou não coopera (0) com outros municípios

"a" : constante da equação

a : coeficientes das variáveis métricas

Ơ : coeficientes das variáveis não métricas

BASE_TRIB : volume de recursos obtidos via receitas tributárias

STRESS : dívida fundada a longo prazo

DEP_FIN : montante de receitas recebidas por meio de transferências intergovernamentais

COND_ECO : PIB per capita

PORTE : população absoluta do município

AGLOM : variável dicotômica que indica se o município pertence (1) ou não (0) à aglomeração urbana

Os estudos de Mazzali e Niero (2015) e Pereira e Moreira (2016b) apontam a regressão logística como técnica ideal para a análise quantitativa, considerando a dicotomia da variável dependente. A análise de metaregressão de Bel e Warner (2016) em 35 artigos empíricos internacionais observam tipicamente o uso da análise multivariada de dados, majoritariamente a regressão logística, para analisar variáveis que explicam os fatores que influenciam a cooperação intermunicipal.

Antes da realização da regressão logística, algumas análises foram efetuadas para explorar os dados, como o cálculo da normalidade das variáveis, para garantir a validade das análises posteriores. Inicialmente, fez-se uma análise das variáveis métricas, para verificar as medidas de tendência central e medidas de dispersão. Os resultados indicaram um alto nível de variação dos dados, conforme demonstrado na tabela 1:

Tabela 1 - Medidas de tendência central e dispersão das variáveis métricas com todos os municípios a

\begin{tabular}{|c|c|c|c|c|c|}
\hline Descrigin & BT & EF & DR & $\mathbf{C E}$ & PM \\
\hline Méla & 99 & 36 & 1.791 & 9.606 & 32001 \\
\hline $\begin{array}{l}\text { Eno } \\
\text { Pailfong }\end{array}$ & 7 & 8 & 37 & 445 & 6.042 \\
\hline Mediana & 5 & - & 1.706 & 6919 & 16.467 \\
\hline $\begin{array}{l}\text { Desnio } \\
\text { Paili.e }\end{array}$ & 151 & 174 & 75 & 9533 & 130.171 \\
\hline CV & $153 \%$ & $481 \%$ & $44 \%$ & $99 \%$ & $397 \pi$ \\
\hline Cutose & 65 & 12 & 61 & 35 & 374 \\
\hline Ass-inetia & 7 & 10 & 6 & 5 & 19 \\
\hline $\mathbf{N}$ & 446 & 458 & 458 & 458 & 458 \\
\hline
\end{tabular}

Fonte: Elaborado pelos autores (2017)

a. Sigla das Variáveis: BT - Base Tributária; EF - Estresse Fiscal; DR -

Dependência de Recursos; CE - Condição Econômica; PM - Porte do Município).

A análise do Coeficiente de Variação (CV) permite inferir se há um alto grau de dispersão nos dados na população observada. Segundo Fávero et al. (2009), um CV superior a 30\% indica que os dados são altamente dispersos, prejudicando as análises estatísticas que dependam da normalidade. Percebeu-se que apenas a Dependência de Recursos aproxima-se desse parâmetro, ainda que o tenha superado. No extremo, o Estresse Fiscal $(\mathrm{CV}=481 \%)$ e o Porte do Município $(\mathrm{CV}=397 \%)$ são as variáveis que apresentam a maior variabilidade. Diante disso, e considerando que o porte é um dos fatores que não somente influencia a motivação para a cooperação, como também as demais variáveis explicativas (Mazzali \& Niero, 2015), optouse por estratificar os municípios de acordo com o porte, utilizando a distribuição de frequência, via software IBM SPSS ${ }^{\curvearrowleft}$, pelo critério de Tukey's Hinges, conforme a Tabela 2: 
Tabela 2 - Distribuição dos municípios da base de dados conforme seu porte

\begin{tabular}{|c|c|c|}
\hline Pante & $\begin{array}{c}\text { Qtde de } \\
\text { Minnigios }\end{array}$ & $\boldsymbol{x}$ \\
\hline Até 11.07n habitantes & 114 & $\mathbf{2 5}$ \\
\hline De 11.078 a 16.466 habitantes & 115 & $\mathbf{2 5}$ \\
\hline De 11.078 a 16.466 habitantes & 115 & $\mathbf{2 5}$ \\
\hline De 11.078 a 16.466 habitantes & 115 & $\mathbf{2 5}$ \\
\hline Total & 458 & 100 \\
\hline
\end{tabular}

Fonte: Elaborado pelos autores (2017).

Com esta distribuição aplicada à variável Porte dos Municípios, analisando os gráficos Boxplots por faixa, percebeu-se uma distribuição normal dos dados, exceto em relação à última faixa (Acima de 26.577 habitantes), na qual o CV foi de 597\%. Nessa faixa foram detectados alguns outliers (municípios de Salvador, Feira de Santana, Vitória da Conquista e Camaçari, inclusive, todos que não haviam celebrado acordo de consórcio intermunicipal). Inicialmente, como feito por Mazzali e Niero (2015, p. 72), optou-se por excluir da base a capital do estado, com o argumento de que na capital há um "enorme distanciamento em termos de população, renda e características sociais com relação aos demais municípios”.

Novas análises exploratórias dos dados do porte foram realizadas e, com a exclusão (um a um, ou seja, retirava-se o maior valor extremo e refazia-se a análise) desses quatro municípios, o CV chegou ao patamar de $64 \%$, ainda elevado. Neste estágio, ainda apareciam como outliers, começando do mais para o menos distante da média, os municípios de Itabuna, Juazeiro, Ilhéus, Lauro de Freitas, Jequié, Alagoinhas, Teixeira de Freitas, Barreiras, Porto Seguro, Simões Filho, Paulo Afonso e Eunapólis. Esses também foram excluídos da base até que se chegou a um CV em torno de 36\%, quando o gráfico Bloxpots apontou três valores extremos (municípios com menos de 100.000 habitantes). Nesse momento, seguiu-se à análise das outras variáveis métricas, sem excluir outros municípios.

Esta análise, por sua vez, demonstrou um alto grau de variabilidade $(\mathrm{CV})$ em relação às outras variáveis. Nesse sentido, adotou-se o processo de normalização das variáveis via transformação para o log base 10 . Após a realização desse processo, na análise das medidas de dispersão, apenas a variável Estresse Fiscal apresentou um CV acima de 30\%. Recorreu-se então a outros testes necessários à validação do modelo de regressão, como exposto na sequência. Mazzali e Niero (2015), tratando do problema de normalidade das variáveis, consideraram que o melhor método de transformação da variável Estresse Fiscal é o da raiz cúbica. Neste estudo, tal procedimento resultou em dados mais dispersos do que a transformação com base no log de 10 . Assim, decidiu-se por excluir da base dois municípios que não haviam celebrado consórcios e apresentavam valor extremo para esta variável. Ainda assim, o CV dessa variável, apesar de ter melhorado, ficou em torno de 60\%, conforme demonstra a Tabela 3. 
Tabela 3 - Medidas de tendência central e de dispersão das variáveis métricas por classe de Porte de Municípios a

\begin{tabular}{|c|c|c|c|c|c|}
\hline $\begin{array}{c}\text { Porte do } \\
\text { Município } \\
\end{array}$ & $\begin{array}{c}\text { Variáveis } \\
\text { b }\end{array}$ & $\mathrm{N}$ & Média & \begin{tabular}{|l|} 
Desvio \\
Padrão \\
\end{tabular} & $\mathrm{CV}$ \\
\hline \multirow{5}{*}{$\begin{array}{l}\text { A te } 11.845 \\
\text { habitantes }\end{array}$} & BT & 108 & 1,71 & $\begin{array}{r}0,32 \\
\end{array}$ & $18,92 \%$ \\
\hline & ES & 45 & 1,20 & 0,89 & $73,95 \%$ \\
\hline & $\mathrm{DR}$ & 108 & 3,31 & 0,10 & $2,98 \%$ \\
\hline & $\mathrm{CE}$ & 113 & 3,88 & 0,20 & $5,25 \%$ \\
\hline & PM & 113 & 3,91 & 0,12 & $3,11 \%$ \\
\hline \multirow{5}{*}{$\begin{array}{c}\text { De } 11.846 \text { a } \\
17.987 \\
\text { habitantes }\end{array}$} & $\mathrm{BT}$ & 112 & 1,76 & 0,27 & $15,40 \%$ \\
\hline & $\mathrm{ES}$ & 40 & 1,19 & 0,69 & $58,45 \%$ \\
\hline & DR & 112 & 3,26 & 0,08 & $2,42 \%$ \\
\hline & $\mathrm{CE}$ & 115 & 3,83 & 0,16 & $4,17 \%$ \\
\hline & PM & 115 & 4,14 & 0,05 & $1,14 \%$ \\
\hline \multirow{5}{*}{$\begin{array}{c}\text { De } 17.988 \text { a } \\
32.300 \\
\text { habitantes }\end{array}$} & $\mathrm{BT}$ & 111 & 1,74 & 0,26 & $14,84 \%$ \\
\hline & $\mathrm{ES}$ & 50 & 1,17 & 0,71 & $61,00 \%$ \\
\hline & DR & 111 & 3,22 & 0,05 & $1,70 \%$ \\
\hline & $\mathrm{CE}$ & 114 & 3,89 & 0,19 & $4,95 \%$ \\
\hline & PM & 114 & 4,32 & 0,06 & $1,44 \%$ \\
\hline \multirow{5}{*}{$\begin{array}{c}\text { A cima de } \\
32.300 \\
\text { habitantes }\end{array}$} & $\mathrm{BT}$ & 96 & 1,99 & 0,35 & $17,51 \%$ \\
\hline & ES & 47 & 1,21 & 0,82 & $67,69 \%$ \\
\hline & DR & 96 & 3,20 & 0,12 & $3,88 \%$ \\
\hline & $\mathrm{CE}$ & 97 & 3,98 & 0,27 & $6,81 \%$ \\
\hline & $\mathrm{PM}$ & 97 & 4,62 & 0,14 & $3,11 \%$ \\
\hline
\end{tabular}

Fonte: Elaborado pelos autores (2017). a. Variáveis transformadas para Log de Base 10

b. Sigla das Variáveis: BT - Base Tributária; EF - Estresse Fiscal; DR -

Dependência de Recursos; CE - Condição Econômica; PM - Porte do Município

Como a dispersão da variável Estresse Fiscal tem fundamento nos aspectos conjunturais que são explicados adiante nas análises de resultados, optou-se então por analisar outros pressupostos da regressão logística para verificar a adequação do modelo, com todas as variáveis propostas por Mazzali e Niero (2015). A análise é apresentada, com os respectivos procedimentos metodológicos estatísticos, na seção seguinte, pelo modelo de regressão. $\mathrm{Na}$ regressão logística, assim como na linear, é possível adotar vários métodos para a inclusão das variáveis no modelo (Field, 2009). Segundo este autor, em estudos nos quais se pretende testar teorias, o método mais indicado é o enter, no qual todas as variáveis são forçadamente incluídas no modelo. Esse foi o método adotado neste estudo, conforme a figura 3.

O teste de Hosmer e Lemeshow analisa se o modelo proposto apresenta diferenças entre o que é observado dos dados e o que ele prevê. Nesse caso, é possível verificar um valor não significativo ( $\mathrm{Sig}>0,05$ ), o que rejeita a hipótese de diferença, ou seja, esse é um indício de que o modelo é um bom previsor dos valores observados (Field, 2009). Por outro lado, a estatística de verossimilhança-log (-2 Log likelihood) é alta, o que para esse autor é um indício de que o modelo pode apresentar uma baixa capacidade explicativa, dito de outro modo, ainda que não apresente diferenças quanto ao que é observado, a capacidade explicativa é pequena. 


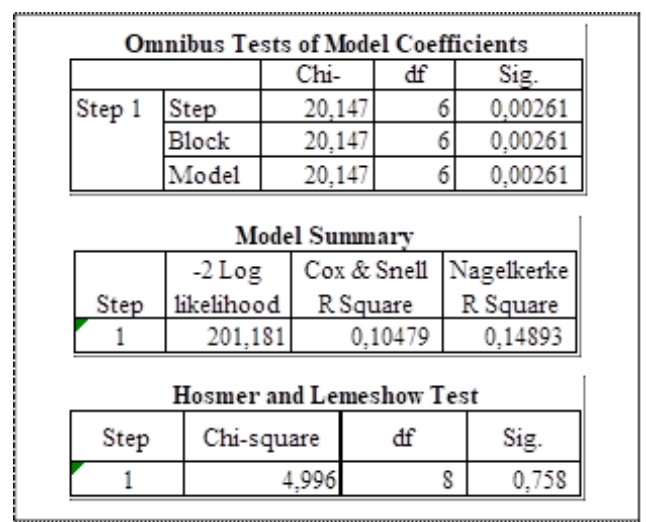

Figura 1 - Saídas: Análise de adequação do Modelo de Regressão Logística Fonte: Elaborado pelos autores (2017).

Não obstante, Corrar, Paulo e Dias Filho (2007) afirmam que o entendimento sobre um valor alto só deve ser considerado quando se parte de uma referência, por exemplo, comparando-se com outro modelo de predição. Conforme sugere Field (2009), a expectativa é que o valor da estatística de verossimilhançalog diminua. De todo modo, o Qui-quadrado do modelo, medido pelo Omnibus Tests, indica que há pelos menos uma das variáveis explicativas que contribui para a predição (Sig. < 0,05).

Apesar de Mazzali e Niero (2015, p. 78) não apresentarem os resultados da estatística de verossimilhançalog, os seus achados com base na análise do $\mathrm{R}^{2}$ (R Square) das estatísticas Cox\&Snell e Nagelkerke indicam o baixo poder explicativo, como também é visto nos dados deste trabalho. Segundo os autores, o modelo utilizado também não pode ser explicativo pois “[...] os valores das estatísticas de $\mathrm{R}^{2}$ são relativamente baixos, sinalizando que há outras variáveis que influenciam a motivação/interesse a cooperar, não consideradas neste estudo". Os achados apresentados, portanto, contribuem para essa constatação ao considerar todas as variáveis do modelo de regressão logística.

Como já visto, alguns procedimentos foram realizados no sentido de reduzir os efeitos da dispersão dos dados. Assim sendo, verificou-se também os pressupostos da regressão logística considerando esses estratos. Todavia, tal tratamento não melhorou o cenário em relação às medidas acima apresentadas, o que levou à promoção de análises para os dados da população de forma conjunta, conforme relatado na seção 4 .

\section{ANÁLISES E RESULTADOS}

Ainda que outras variáveis sejam necessárias para explicar a decisão de cooperação intermunicipal, como em Mazzali e Niero (2015), optou-se por verificar a relação das variáveis consideradas neste modelo com essa decisão de cooperação. Nesse sentido, em relação aos municípios observados, o modelo tem um nível de acerto, em geral, de 74,7\% e, quando se analisa apenas os municípios que cooperaram, consegue acertar em $96,9 \%$ dos casos, conforme demonstrado na figura 3: 


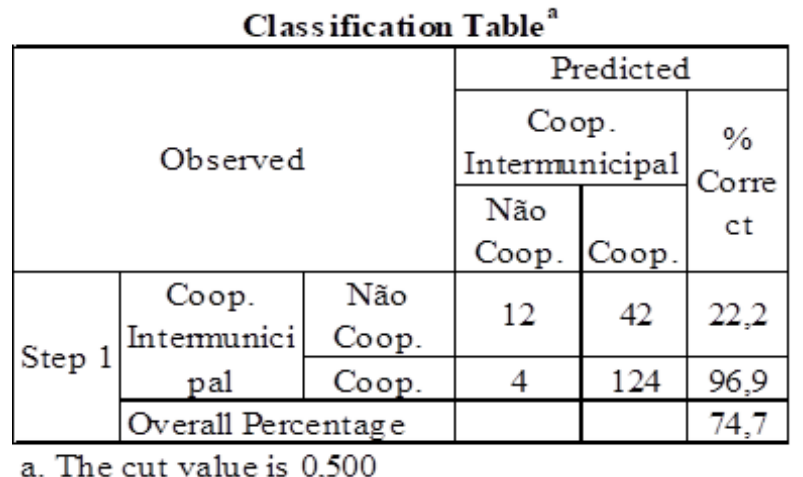

Figura 3 - Saída 2: Nível de acerto do Modelo de Regressão Logística Fonte: Elaborado pelos autores (2017).

Outra análise do modelo de regressão verifica a significância das variáveis individualmente. Em particular, a estatística Wald é um importante indicador (Field, 2009; Corrar, Paulo \& Dias Filho, 2011). Os dados podem ser observados na figura 4:

\begin{tabular}{|c|c|c|c|c|c|c|c|c|}
\hline \\
\hline & & \multirow[b]{2}{*}{ B } & \multirow[b]{2}{*}{ S.E. } & \multirow[b]{2}{*}{ Wald } & \multirow[b]{2}{*}{ Sig. } & \multirow[b]{2}{*}{$\operatorname{Exp}(B)$} & \multicolumn{2}{|c|}{$95 \%$ C.I. EXP(B) } \\
\hline & & & & & & & Lower & Upper \\
\hline \multirow{7}{*}{$\begin{array}{c}\text { Step } \\
1\end{array}$} & BT & $-1,69$ & 0,76 & 4,9610 & 0,026 & 0,185 & 0,042 & 0,817 \\
\hline & ES & 0,07 & 0,22 & 0,1045 & 0,747 & 1,075 & 0,694 & 1,664 \\
\hline & DR & $-4,37$ & 2,79 & 2,4500 & 0,118 & 0,013 & 0,000 & 3,013 \\
\hline & $\mathrm{CE}$ & 1,00 & 0,98 & 1,0373 & 0,308 & 2,724 & 0,396 & 18,733 \\
\hline & PM & $-1,54$ & 0,91 & 2,8800 & 0,090 & 0,214 & 0,036 & 1,270 \\
\hline & $\mathrm{AU}$ & $-2,64$ & 1,31 & 4,0238 & 0,045 & 0,072 & 0,005 & 0,941 \\
\hline & Const & 23,27 & 11,31 & 4,2346 & 0,040 & \multicolumn{2}{|c|}{12796276280.458} & \\
\hline
\end{tabular}

Figura 4 - Saída 3: Modelo de Regressão com as variáveis propostas

Fonte: Elaborado pelos autores (2017). a. Sigla das Variáveis: BT - Base Tributária; EF - Estresse

Fiscal; DR - Dependência de Recursos; CE - Condição Econômica; PM - Porte do Município.

Ressalta-se que essas variáveis estão transformadas em logaritmos de base 10. Percebe-se que apenas as variáveis "Base Tributária" e "Aglomeração Urbana” apresentam-se estatisticamente significativas (Nível de Confiança de 95\%). Esta última também foi significante para Mazzali e Niero (2015). Esses autores consideraram um nível de significância de $10 \%$, o que, se assim fosse considerado neste estudo, faria do porte dos municípios (medido pelo número de habitantes) uma variável significativa para o modelo, da mesma forma que no estudo deles. As análises da estatística $\operatorname{Exp}(\mathrm{B})$ e dos intervalos de confiança confirmam esse entendimento.

Optou-se por analisar o modelo de regressão excluindo as variáveis que não apresentavam significância, começando pelo Estresse Fiscal, em função de seu Sig. (0,7466) e do fato de essa variável ter apresentado alto $\mathrm{CV}$, conforme já demonstrado. A exclusão apenas da variável Estresse Fiscal não melhorou as estatísticas do modelo, pelo contrário, o resultado da estatística de verossimilhança-log, por exemplo, foi de 499,468, comparativamente pior do que o modelo com todas as variáveis. Logo, um novo teste foi aplicado, excluindo também a variável Condição Econômica (Sig. 0,3084) e, posteriormente, a Dependência de Recursos (Sig. 0,1175). Novamente, os resultados não foram estatisticamente melhores, adotando então a análise com todas as variáveis, como exposto a seguir.

Os coeficientes das variáveis (B) estão na base $\log 10$, assim, é preciso ajustá-las para interpretar estes sinais. Nesse sentido, foram calculados os antilogaritmos desses coeficientes por meio da exponenciação à base 10. Para as três variáveis, os resultados são: 0,0206 para a variável Base Tributária, 0,0286 para o Porte 
dos Municípios e 0,0023 para a Aglomeração Urbana. Assim, é possível observar que essas três variáveis aumentam a possibilidade de os municípios baianos celebrarem acordos de cooperação intermunicipal.

Como o resultado foi positivo para a variável base tributária, relativa aos recursos de receita tributária, a hipótese 1 foi confirmada. Ou seja, existe relação positiva entre recursos fiscais e a cooperação nos municípios baianos, em conformidade com os achados de Mazzali e Niero (2015) para o estado de São Paulo, que apontam que quanto maior a receita tributária do município, maior a probabilidade de ele celebrar acordos de cooperação com outros municípios, coadunando também com os demais estudos em cooperação intergovernamental (Bel \& Warner, 2016; Krueger \& Mcguire, 2005; Leroux \& Carr, 2007). Em contraponto, o estudo de Pereira e Moreira (2016a) evidencia a influência negativa da base tributária na formação de consórcios públicos municipais a nível nacional.

Considerando as variáveis da regressão relacionadas com as hipóteses 2, 3 e 4, não foi possível realizar inferências estatísticas a partir do modelo proposto nesse estudo. Quanto à hipótese 2 relacionada ao nível de estresse fiscal, em específico, no caso dos consórcios de desenvolvimento do estado da Bahia foram levantadas duas reflexões que auxiliam na compreensão do resultado: (i) Há uma dificuldade de muitos dentre os pequenos municípios brasileiros em contraírem empréstimos; (ii) o que faz com que em muitos municípios baianos o valor dessa variável seja zero (gerando problemas de normalidade). Logo, o uso da dívida fundada per capita como indicador pode não ser suficiente para explicar o estresse fiscal, conforme sugerido pela literatura.

O estudo de Andrew (2009) trata dessa segunda reflexão. Apesar de a variável Estresse Fiscal ser frequentemente utilizada em alguns estudos empíricos como motivadora da cooperação por parte de governos locais, ela apresenta um problema significativo pelo fato de não terem desenvolvido ainda medidas adequadas dos conceitos que compóem o estresse fiscal em estudos transversais. Por outro lado, o Brasil é mais complexo que outros países, devido às heterogeneidades espacial, política, social e econômica (Rios, 2014). Particularmente, as heterogeneidades das capacidades, estruturas administrativas e questões de agência dos municípios brasileiros podem impactar na obtenção de recursos adicionais via empréstimos (dívida fundada per capita) por intermédio de projetos e programas de financiamento de outras esferas federativas. Esta limitação é reforçada pelo art. 167, III da Constituição Federal (Brasil, 1988), que veda a obtenção de crédito que exceda o montante das despesas de capital. Os dados apontam que grande parte dos municípios baianos de menor porte não alcançaram empréstimos de outras esferas federativas ou de outras fontes como, por exemplo, do Banco Interamericano de Desenvolvimento (BID).

Quanto à hipótese 3, sobre a dependência de recursos das esferas superiores, parecem ser latentes os efeitos da diversidade e heterogeneidades econômicas e sociais no território brasileiro, haja vista o caso de São Paulo. Nesse estado, há muitos anos houve algumas iniciativas de cooperação induzida por esferas superiores que produziram grandes receitas de transferências de esferas superiores (Mazzali \& Niero, 2015). No caso da Bahia, a diversidade em termos de porte, renda per capita, dimensões geográficas e demográficas carece de um estudo mais aprofundado.

A hipótese 4 também não foi possível analisar, pois o resultado da determinante não foi significante. Segundo Andrew (2009, p. 137), a variável PIB per capita, embora seja uma convenção na literatura, parece não ser um indicador ideal para a motivação à cooperação na população estudada, visto que "as pesquisas empíricas mostram que a realidade é mais complexa do que essa proposição sugere”. O autor também afirma que a relação entre PIB per capita e a adoção de cooperação intergovernamental é "possivelmente não-linear, o que significa que ambos os achados estão provavelmente corretos".

Já a hipótese 5 foi confirmada, conforme os achados de Mazzali e Niero (2015), ou seja, há uma associação positiva entre o porte do município e a decisão em cooperar por meio de consórcios. Em contrapartida, essa associação é negativa nos resultados de Bel e Warner (2016) e Pereira e Moreira (2016a). Particularmente no Brasil, o estudo de Pereira e Moreira (2016) no âmbito nacional, aponta que quanto menor o porte do município, maior o interesse na participação em acordos de desenvolvimento. 
Concluindo a análise, a partir dos dados resultantes da regressão logística, foi possível confirmar a hipótese 6, assim como no estudo de Mazzali e Niero (2015). Logo, quando um município faz parte de uma aglomeração urbana, ele está mais propenso a estabelecer acordos de cooperação intermunicipal em virtude do aumento da probabilidade de transbordamentos (Post, 2004) e identificação de problemas semelhantes de desenvolvimento como motivadores da cooperação para o desenvolvimento interlocal. Outro ponto salutar sobre os consórcios de desenvolvimento do estado da Bahia é que alguns deles são multifinalitários. Logo, para aqueles municípios participantes de consórcios que estão situados em aglomerações urbanas, a prestação de serviços multifinalitários tende a produzir maiores economias de escala, motivando-os a constituírem acordos intermunicipais em prol do desenvolvimento (Bel \& Warner, 2016).

\section{CONCLUSÕES}

O objetivo deste artigo foi o de identificar quais características econômicas, fiscais, demográficas e geográficas dos municípios baianos influenciam a formação de consórcios intermunicipais de desenvolvimento. Para o alcance desse objetivo, na identificação dessas características foi estimado um modelo do tipo logit para as 458 observações dos 417 municípios do estado da Bahia. Pela carência de estudos que investiguem os fatores que influenciam a formação de consórcios municipais no âmbito do desenvolvimento, o presente artigo privilegiou os fatores exógenos da perspectiva da Ação Coletiva Institucional como lente de análise das condições fiscais e econômicas e características demográficas e geográficas, com enfoque no interesse em cooperar.

Em relação aos resultados, foram identificadas três características correlacionadas positivamente com e que incentivam a formação de consórcios municipais de desenvolvimento na Bahia: A receita tributária (base tributária), o porte dos municípios (população) e a inserção dos municípios em aglomerações urbanas. A diversidade do território baiano certamente contribui para o resultado encontrado, por ser um estado com extensão territorial expressiva, composto, em contrapartida, por apenas duas regiões metropolitanas e duas aglomerações urbanas que concentram 40,90\% da população nessa área. Assim sendo, os achados deste estudo na Bahia, como os de Mazzali e Niero (2015) em São Paulo, confirmam o efeito transbordamento como influência à cooperação, em que municípios de maior porte e maior receita tributária, pertencentes a aglomerações urbanas, tendem a buscar a cooperação dos municípios vizinhos para que, juntos, resolvam questões comuns da região.

É relevante que novos estudos privilegiem as características sociais, os custos de transação relacionados às barreiras a cooperação, bem como os fatores endógenos no caso da Bahia, visando ampliar o escopo de análise. Nesse sentido, a pesquisa sistemática das motivações e barreiras à cooperação intergovernamental no Brasil mostra-se necessária, face às heterogeneidades do território brasileiro explicitadas e a limitação de não incorporar as barreiras à cooperação neste estudo. Outras dimensões e variáveis precisam ser analisadas para compreender a influência à cooperação, pode ser verificada, por exemplo, a relação entre a decisão de cooperar e a falta de escala, ou de capacidade a nível municipal, para a execução de políticas públicas, como sugerem Linhares et al. (2017).

Embora os estudos internacionais, que utilizam a regressão logística como método quantitativo, encontrem uma significância positiva na influência das restrições fiscais na motivação a cooperar (Bel \& Warner, 2016), os estudos brasileiros, considerando este, ainda não chegaram a esse consenso. Isso pode ser em função da utilização da métrica dívida pública para captar os dados da variável Estresse Fiscal, pois essa métrica apresenta problemas inerentes ao contexto brasileiro, como já explicitado neste estudo. Uma solução, conforme apontado por Hawkins (2007), é captar o estresse fiscal por meio da relação entre a carga tributária e o volume de políticas públicas adotadas pelo município.

Numa sociedade complexa, certamente há inúmeras variáveis que influenciam as decisões. Por exemplo, Randolph e Magalhães (2017) demonstram como a decisão de formação do Consórcio Intermunicipal de 
Desenvolvimento do Leste Fluminense (CONLESTE) foi influenciada pela decisão da Petróleo Brasileiro S.A. (Petrobras) de montar um complexo petroquímico naquela região. Esse estudo, portanto, não quis esgotar todas as variáveis capazes de influenciar a cooperação, mas sim testar a relação entre algumas variáveis apontadas pela teoria e a decisão dos gestores de municípios baianos. Todavia, essas outras aqui apontadas podem compor novas agendas de pesquisa.

Ainda em relação à formação dessa agenda, existem algumas dimensões relevantes como a avaliar a eficácia (ou não) da cooperação intermunicipal em termos de economia de escala (Bel \& Warner, 2016). Andrew (2009) afirma a conformação da literatura quanto à obtenção de economias de escala pelos governos locais quando cooperações interlocais envolvem alto montante de capital inicial, porém denuncia que poucos pesquisadores se dedicam a investigar quais são os resultados associados.

Além dessas, as pesquisas futuras devem se dedicar a verificar fatores endógenos que influenciam ou não os municípios a participarem de consórcios intermunicipais como já apontado por estudiosos. Nesse sentido, novas pesquisas podem avaliar se a orientação política exerce influência na cooperação, pois esse aspecto é pouco observado nos estudos e ainda não evidencia um possível fator determinante na cooperação municipal (Bel \& Warner, 2016; Mazzali \& Niero, 2015).

\section{REFERENCIAS}

Abrucio, F. L. (2005). Reforma do Estado no Federalismo Brasileiro: a situação das administrações públicas estaduais. Revista Brasileira de Administração Pública, 39(2), 401-420.

Abrucio, F. L. \& Sano, H. A. (2011). Experiência de Cooperação Interestadual no Brasil: formas de atuação e seus desafios. Cadernos ADENAUER, 4, 22-31.

Abrucio, F. L.; Filippim, E. S. \& Dieguez, R. C. (2014). Inovação na cooperação intermunicipal no Brasil: a experiência da Federação Catarinense de Municípios (Fecam) na construção de consórcios públicos. Revista de Administração Pública, 47(6), 1543-1568.

Afonso, A. S.; Vieira, C. M.; Santos Júnior, J. L. \& Doin, T. A. F. (2016). Desafios para o financiamento dos planos municipais de resíduos sólidos: O caso do Condoeste. In: J. L. dos Santos Júnior \& A. S. Afonso. (Orgs.). Desafios para o desenvolvimento capixaba: uma perspectiva transdisciplinar. Curitiba: CRV.

Andrew, S. A. (2009). Recent Developments in the Study of Interjurisdictional Agreements: An Overview and Assessment. State and Local Government Review, 41(2), 133-142.

Bel, G. \& Warner, M. E. (2016). Factors explaining inter-municipal cooperation in service delivery: a meta-regression analysis. Journal of Economic Policy Reform, 19(2), 91-115.

Benton, J. E. (2013) Local Government Collaboration: Considerations, Issues, and Prospects. State and Local Government Review, 45(4), 220-223.

Brasil. Constituição Federal de 1988. Promulgada em 5 de outubro de 1988. Disponível em http:// www.planalto.gov.br/ccivil_03/constituicao/constituição.htm. Acesso em 10/12/2017.

Carvalho, S. L. De; Xaxier, T. Dos S. \& Pinto, F. L. B. (2016). Trajetória dos consórcios públicos baianos: oportunidades e desafios para uma política de desenvolvimento territorial. Anais do IX Congresso CONSAD de Gestão Pública, Brasília, DF, Brasil, 1-27.

Cruz, M. do C. M. T.; Araújo, F. F. \& Batista, S. (2011). Consórcios numa perspectiva histórico-institucional. In: Municípios e estados: experiências com arranjos cooperativos. Rio de Janeiro: Fundação Konrad Adenauer.

Cunha, R. E. da. (2004). Federalismo e relações intergovernamentais: os consórcios públicos como instrumento de cooperação. Revista do Serviço Público, 55(3), 5-36.

Farah, M. F. S. \& Jacobi, P. (2000). Governos locais e cooperação inter e intragovernamental no Brasil. In: M. F. Santos \& H. B. Barbosa (Eds.). Novas experiências de gestão pública e cidadania. São Paulo: FGV.

Fávero, L. P (2015). Análise de dados: modelos de regressão com Excel' ${ }^{\circ}$, Stata e ${ }^{\circ}$ SPS ${ }^{\circ}$. Rio de Janeiro: Elsevier.

Feiock, R. C. (2007). Rational Choice and Regional Governance. Journal of Urban Affairs, 29(1), 47-63. 
Feiock, R. C. (2013) The institutional collective action framework. Policy Studies Journal, 41(3), 397-425.

Feiock, R. C. \& Carr, J. B. (2001). Incentives, entrepreneurs, and boundary change: A Collective Action Framework. Urban Affairs Rewiew, 36(3), 382-405.

Galindo, J. M.; Cordeiro, J. C.; Villani, R. A. G.; Barbosa, E. A., Filho \& Rodrigues, C. S. (2014). Gestão interfederativa do SUS\#: a experiência gerencial do Consórcio Intermunicipal do Sertão do Araripe de Pernambuco. Revista de Administração Pública, 48(6), 1545-1566.

Grin, E. J.; Segatto, C. I. \& Abrucio, F. L. (2016). El asociativismo intermunicipal en Brasil. (1a ed). In. D. Cravacuore \& A. Chacón (Eds.). El Asociativismo Intermunicipal en América Latina. Santiago do Chile: Universidad Tecnológica Metropolitana.

Hawkins, C. V. (2007). Local government joint ventures: cooperation and competition for economic development (Tese de doutorado). Department of Urban and Regional Planning of the Florida State University, Tallahassee.

Krueger, S. \& Mcguire, M (2005). A Transaction Costs Explanation of Interlocal Government Collaboration. Michigan: Wayne State University.

Leroux, K. E \& Carr, J. B (2007). Explaining local government cooperation on public works: evidence from Michigan. Public Works Management and Policy, 12(1), 344-358.

Linhares, P. de T. F. S.; Messenberg, R. P. \& Ferreira, A. P. L. (2017). Transformações na Federação Brasileira: o consórcio intermunicipal no Brasil do início do século XXI. Boletim de Análise Político-Institucional do IPEA - Instituto Brasileiro de Pesquisas Aplicadas, 12.

Machado, J. A. \& Andrade, M. L. C (2014). Cooperação intergovernamental, consórcios públicos e sistemas de distribuição de custos e benefícios. Revista Administração Pública, 48(3), p. 695-720.

Mazzali, L. \& Niero, J. C. C. (2015). Arranjos intermunicipais de desenvolvimento: Fatores condicionantes da propensão a cooperar. Revista Brasileira de Gestão e Desenvolvimento Regional, 11(2), 60-92.

Morgan, D. R. \& Hirlinger, M. W. (1991). Intergovernmental service contracts: A multivariate explanation. Urban Affairs Quarterly, 27(1), 128-144.

Nascimento, A. M. \& Fernandes, A. S. A. (2015). Relações de Cooperação em Consórcios Públicos de Regiões Metropolitanas: análises do CONDIAM / PB e Consórcio Grande Recife / PE. Teoria e Prática em Administração, 5(1), 25-52.

Oliveira, F. de. (1995). A crise da federação\#: da oligarquia à globalização. In: R. B. A. Afonso \& P. L. B. Silva (Eds.). A federação em perspectiva. São Paulo: Fundação do Desenvolvimento Administrativo.

Pereira, G. A. \& Moreira, T. B. da S. (2016b). A influência dos consórcios intermunicipais de saúde no índice Firjan de desenvolvimento municipal (IFDM). Planejamento e Políticas públicas, 46(1), 131-159.

Pereira, G. A. \& Moreira, T. B. da S. (2016a). Consórcio Público Intermunicipal: características dos municípios participantes. Revista de Políticas Públicas, 20(1), 307-325.

Post, S. S. (2004). Metropolitan area governance and institutional colletive action. In: R. C. Feiock (Ed.). Metropolitan governance: conflit, competition, and cooperation. Washington, D.C.: Georgetown University Press, 67-93.

Randolph, R. \& Magalhães, G. A. (2017). Governança territorial e seus desafios: Reflexões sobre consórcios intermunicipais e comitês hidrográficos. Brazilian Journal of Development, 3(especial), 389-405.

Rios, R. B. (2015). A dimensão político-territorial dos consórcios públicos intermunicipais: uma análise da espacialidade no contexto brasileiro. Anais do I Congresso Brasileiro de Geografia Política, Geopolítica e Gestão do Território, Porto Alegre-RS, Brasil.

Tavares, A. F. \& Feiock, R. C. (2014). Intermunicipal Cooperation and Regional Governance in Europe: An Institutional Collective Action Framework. Anais do World CongressInternational Political Science Association. Montreal.

Teixeira, E. C. (1999). Sociedade Civil e seu Papel Político: o local e o global como espaços de participação cidadã. Organizações \& Sociedade, 6(14), 105-114.

\section{BY-NC-ND}

